\section{cAMPing out}

\section{Leland Hartwell}

CELLS often exhibit a remarkable ability to recover from insults, hinting at the existence of homeostatic mechanisms that return them to an equilibrium state. This point was powerfully demonstrated almost 40 years ago. When a portion of the cytoplasm of a growing amoeba was repeatedly cut from the cell, the cell continued accumulating mass, but never divided - apparently it was aware that it had not reached a size sufficient for division.

Two reports on pages 339 and 342 of this issue ${ }^{2,3}$ uncover a new role for cyclic AMP in the homeostatic mechanism that integrates cell growth with division. They deal with the budding yeast, Saccharomyces cerevisiae, which has been a popular organism for studies on the relationship between growth and division in part because the new growth in each cell cycle is visible as a distinct body, the bud, that becomes the daughter cell.

A mother cell begins the cell cycle in $\mathrm{G} 1$ phase; it produces a new bud concomitant with $\mathrm{S}$ phase; the bud grows throughout $\mathrm{S}$ and $\mathrm{G} 2$; the nucleus divides at $\mathrm{M}$ with one nucleus going to the mother and one to the bud, and the cell then divides (see figure). Growth is integrated with division in G1. This is most evident from the different behaviours of the mother cell and daughter bud in the next cell cycle. The daughter is normally smaller than the mother cell at division, especially under conditions of slow growth. The daughter cell delays more than the mother in the G1 phase of the next cell cycle before producing a bud, giving itself time to grow to the size characteristic of a mother cell ${ }^{4}$. There is no requirement for growth in other phases of the cell cycle because, if growing cells are suddenly starved of nitrogen to prevent protein accumulation, all cells that have budded complete the cell cycle and divide without net growth ${ }^{5}$. Buds that were small at the time of starvation produce tiny cells which, upon readdition of nitrogen, stay in G1 until they have reached the characteristic size of mother cells.

These observations suggest that cells must simply accumulate enough stable protein in order to pass from $\mathrm{G} 1$ to $\mathrm{S}$. Others, however, indicate that the control is more dynamic, responding to the rate as well as the amount of growth. If the rate of protein synthesis is limited in specific ways, for example, then cells can greatly exceed the critical size that normally char-

acterizes the G1/S transition ${ }^{6}$. Moreover, cells have characteristically different sizes with different carbon sources and, when G1 cells are shifted from one carbon source to another, they adjust to the new size requirement for budding before moving on to $\mathrm{S}$ phase ${ }^{7}$. From these findings it would seem that the critical component monitoring growth is unstable.

Molecular definition of the homeostatic mechanism that integrates growth with

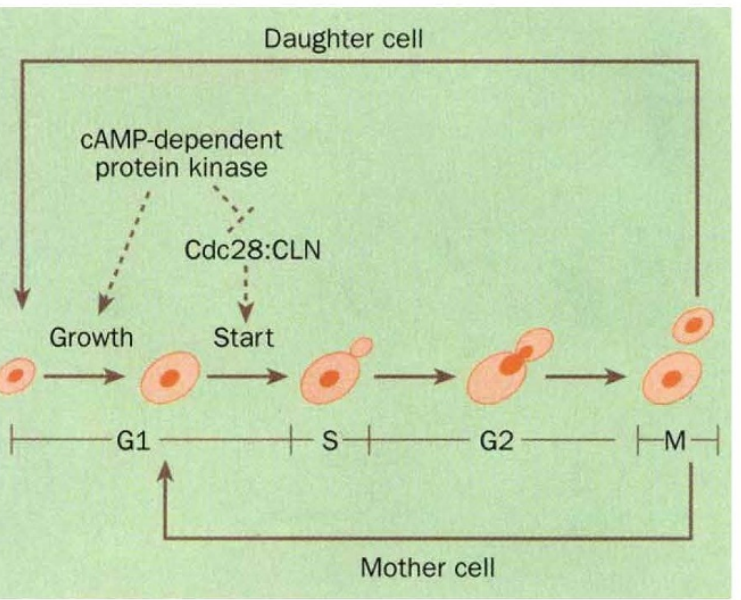

The cell cycle consists of G1, S, G2 and M phases. In Saccharomyces cerevisiae, growth is coordinated with division in the G1 interval. Smali daughter celis, produced under conditions of growth limitation, do not pass Start until they have achieved the size of mother cells. cAMP-dependent kinases have antagonistic roles at Start, being required for owth and inhibiting the transcription of cyclins.

division will require knowledge of the molecular components that mediate 'growth' and 'division', respectively, and those that communicate between them. Previous work implicated the cAMPdependent protein kinases as important mediators of growth, and the cyclindependent kinases as mediators of division. The two new papers ${ }^{2,3}$ provide a link between them. A variety of mutations that inactivate either the production of CAMP or their targets, the cAMP-dependent protein kinases, result in a cessation of growth and arrest of the cell cycle in G1 (ref. 8). Mutations that inactivate the $S$. cerevisiae cyclin-dependent kinase $\mathrm{Cdc} 28$ arrest the division cycle in G1 while growth continues ${ }^{9}$. The G1 collaborators of the cyclin-dependent kinases, the cyclins CLN1, CLN2 and CLN3, are metabolically unstable, being synthesized in G1 and degraded at the G1/S boundary. The cyclins are therefore prime candidates for the dynamic component monitoring size control; indeed, cyclin deficiency can produce larger than normal cells ${ }^{10}$ and cyclin excess smaller than normal cells ${ }^{11}$.

Baroni et al. ${ }^{2}$ and Tokiwa et $a l^{3}$ use different approaches to come to the same conclusion - that cAMP-dependent protein kinases inhibit transcription of the CLN1 and CLN2 genes. Moreover, although transcription of $C L N 3$ is not subject to this inhibition, it may play a special role in mediating the integration of division and growth through the products of $C L N 1$ and $C L N 2$. The addition of cAMP to the culture medium of cells under special physiological conditions or for cells altered in cAMP uptake or metabolism results in G1 arrest, growth that exceeds the normal size at budding, and inhibition of $C L N 1$ and $C L N 2$ transcription. Expression of $C L N 1$ or $C L N 2$ in these cells under the control of a promoter not subject to cAMP regulation overcomes the G1 arrest, showing that $\mathrm{G} 1$ arrest results from failure of CLN1 or CLN2 protein to accumulate. Mutations in the regulatory subunit of the cAMP-dependent kinases or in the kinases themselves demonstrate that these effects are being mediated through the cAMP-dependent kinases. Hence these experiments show that cAMP exercises negative control on division through the cyclins, whereas previous experiments, mentioned above, demonstrated that it has positive control on both growth and division.

How growth and division are coordinated through these antagonistic effects remains to be seen. One clue comes from work showing that reducing the rate of initiation of polypeptide chains can arrest cells in G1, in spite of the fact that the cells grow larger than the unperturbed G1 cell, whereas limiting the rate of polypeptide elongation does not have this effect $^{6}$. So one important task will be to define the relationship between the initiation of protein synthesis, cAMP metabolism and expression of $C L N$ genes.

Leland Hartwell is in the Department of Genetics, SK-50, University of Washington, Seattle, Washington 98195, USA.

1. Prescott, D. M. Exp/CellRes. 11, 86-98(1956)

2. Baroni, M. D., Monti, P. \& Alberghina, L. Nature 371 339-342 (1994)

3. Tokiwa, G.. Tyers, M., Volpe, T. \& Futcher, B. Nature 371 342-345 (1994)

4. Lord, P. G. \& Wheals, A. E.J. Bact. 142, 808-818 (1980).

5. Johnston, G. C. Pringle, J.R. \& Hartwell. L. H. Exp/Cell Res. 105, 79-98 (1977).

6. Hanic-Joyce, P. J., Johnston, G. C. \& Singer. R. A. Exp/ CellRes. 172, 134-145 (1987).

7. Lorincz, A. \& Carter, B. L. A. J. gen. Microbiol. 113. 287-295 (1979).

8. Matsumoto, K. Uno, I. Oshima, Y. \& Ishikawa, T. Proc. natn. Acad. Sci. U.S.A. 79, 2355-2359 (1982).

. Hartwell, L. H. Culotti, J., Pringle, J R. \& Reid, B. J. Science 183, 46-51 (1974).

10. Richardson, H. E., Wittenberg, C., Cross, F. \& Reed, S. I Cell 59, 1127-1133 (1989)

11. Nash, R., Tokiwa, G., Anand, S., Erickson, K. \& Futcher A. B. EMBO J. 7, 4335-4346 (1988) 